\title{
Application of vitamin A palmitate eye gel and nurse value of Watson's theory of caring in children with dry eye after strabismus surgery: a randomized trial
}

\author{
Suling Yang ${ }^{1 \#}$, Weina Guo ${ }^{2 \#}$, Yuexing Gong ${ }^{3}$, Jiancang Wang ${ }^{1}$, Lu Chen ${ }^{1}$, Jingcong Zhao ${ }^{1}$, Xiting Guo ${ }^{1}$, \\ Jie Bai ${ }^{2}$, Yanxia Song ${ }^{1}$ \\ ${ }^{1}$ Department of Ophthalmology, Children's Hospital of Hebei Province, Shijiazhuang, China; ${ }^{2}$ Department of Ophthalmology, Logistic Support \\ Forces of the Chinese People's Liberation Army 980 Hospital, Shijiazhuang, China; ${ }^{3}$ Department of Nursing, Shijiazhuang Medical College, \\ Shijiazhuang, China \\ Contributions: (I) Conception and design: S Yang, Y Song, W Guo; (II) Administrative support: J Wang; (III) Provision of study materials or patients: \\ S Yang, W Guo, J Zhao, Y Gong, X Guo; (IV) Collection and assembly of data: S Yang, W Guo, X Guo, J Bai; (V) Data analysis and interpretation: S \\ Yang, Y Song, W Guo, J Wang, L Chen; (VI) Manuscript writing: All authors; (VII) Final approval of manuscript: All authors. \\ \#These authors contributed equally to this work. \\ Correspondence to: Yanxia Song. Department of Ophthalmology, Children's Hospital of Hebei Province, Shijiazhuang 050000, China. \\ Email: songyanxia5056@126.com.
}

Background: After strabismus surgery, the local swelling of conjunctival wound will affect the uniform distribution of tears on the ocular surface, and the inflammatory reaction will affect the stability of tear film, which will easily lead to iatrogenic dry eye. This study aimed to investigate the effect of vitamin A palmitate (VAP) eye gel application in dry eye and the advantages of Watson's theory of caring.

Methods: Two hundred and forty children with dry eye after strabismus surgery treated in our hospital from September 2018 to September 2020 were selected as the study subjects, and were randomly divided into control group and observation group according to the allocation ratio of 1:1. Watson's theory of care nursing was applied in the observation group, and VAP eye gel was additionally dropped into the eye. Mouse conjunctival goblet cells (GCs) were used to detect the effect of VAP on the growth of GCs. Treatment compliance, improvement of dry eye symptoms [Schirmer I test (SIT), tear film break-up time (BUT), and fluorescent staining (FL) score], inflammatory factor levels in tears, clinical efficacy, and parents' satisfaction were compared.

Results: It was found that VAP eye gel could better promote the proliferation of GCs. After nursing and clinical treatment, the dry eye symptoms were improved in all included children, and improvements in the SIT, BUT, and FL scores were more obvious in the observation group. Watson's theory of care nursing could effectively improve the children's treatment compliance and parents' satisfaction.

Conclusions: As a result, the application of VAP eye gel and Watson's theory of care nursing could effectively reduce the occurrence of dry eye after strabismus surgery, and were of great importance for improving the relationship between nurses and patients and building a harmonious hospital.

Trial Registration: Chinese Clinical Trial Registry ChiCTR2100049136.

Keywords: Dry eye; vitamin A palmitate eye gel (VAP eye gel); Watson care theory; postoperative strabismus

Submitted Jul 27, 2021. Accepted for publication Sep 16, 2021.

doi: $10.21037 / \mathrm{tp}-21-385$

View this article at: https://dx.doi.org/10.21037/tp-21-385 


\section{Introduction}

Dry eye is a common disease in ophthalmology, which causes dryness and foreign body sensations as well as burning pain, and can seriously affect the quality of life of patients (1). In recent years, the incidence rate of dry eye has been increasing annually due to the increased utilization rate of electronic terminal products in work and living environments, irregular work schedules, and poor eye hygiene habits. Furthermore, the proportion of children with dry eye is also increasing (2).

As a special group of patients with dry eye, due to their immature mental development (3), the discomfort symptoms caused by dry eye will not only affect the quality of life of children, but also easily lead to emotional fluctuations, anxiety, and depression, which will have adverse effects on their communication in daily life. Specifically, for some children with iatrogenic dry eye after strabismus surgery, the local swelling of the conjunctival wound after strabismus surgery will affect the uniform distribution of tears on the ocular surface (4), and the postoperative inflammatory reaction will affect the stability of the tear film, eventually leading to the occurrence of dry eye (5). Children are easily rejected and alienated by other playmates in life because of strabismus (6); the occurrence of dry eye after surgery will further impact their mentality and follow-up treatment. On the other hand, the treatment of dry eye is a long-term process, and the clinical effect depends on the treatment compliance of children and their families (7). Therefore, exceptional nursing and follow-up during the treatment of children with dry eye after strabismus surgery, as well as the provision of additional care, will play a significant role in improving their treatment compliance and clinical effect.

With the continuous development of nursing science, the level of nursing technology and the types of nursing materials are constantly being updated and improved. At the same time, the concept of nursing is also being continually updated and developed. The patient-oriented nursing concept is constantly injected into the daily service, and aims to provide better quality service for patients. Caring is the core of nursing in Watson's theory of care (8). In the day-to-day nursing process, nurses should consider "respect" as the connotation and "concern" as the driving force, which will play a significant role in helping to build a harmonious medical environment (9), thereby improving patient's medical experience and recovery. Specifically for children with dry eye after strabismus surgery, due to their immature mental development, postoperative dry eyes and burning pain will further deepen their panic and anxiety. Therefore, upholding Watson's "human text" nursing concept and carry out individualized nursing for each child is crucial for clinical treatment and postoperative rehabilitation.

In this study, vitamin A palmitate (VAP) eye gel is applied for the treatment of dry eye in children after strabismus surgery, as surgery will cause adverse effects on the physiological structure of the ocular surface, and destroy the stability of the tear film, which will aggravate the postoperative discomfort because of the lack of tear lubrication (10). Compared with other dry eye drugs, such as sodium hyaluronate and Carbomer, VAP eye gel can not only play a role of replenishing water and lubricating the eyeball, but vitamin A supplementation can improve the dehydration symptoms of corneal and conjunctival epithelial cells, and promote the normal differentiation and proliferation of corneal epithelium and conjunctival epithelium (11). Furthermore, vitamin A can promote GCs to secrete more mucin, so as to maintain the integrity of tear film and make the eyeball get more lubrication. At the same time, studies have shown that modifying VAP with relevant coatings, such as $\mathrm{N}$-trimethyl chitosan, can increase the retention time of VAP in the eye, which can make it continuously released in the eye and improve its bioavailability, so as to be better absorbed by the cornea and conjunctiva (12). Therefore, the aim of this study is to investigate the effect of VAP eye gel on dry eye after strabismus surgery and the advantages of applying Watson's theory of care during the treatment period. We present the following article in accordance with the CONSORT reporting checklist (available at https://dx.doi.org/10.21037/ tp-21-385).

\section{Methods}

\section{Materials}

\section{Research subject selection}

Two hundred and forty children with dry eye treated in our hospital from September 2018 to September 2020 were selected as the study subjects. All children and their guardians who participated in the study gave informed consent to the study and voluntarily signed the informed consent. This prospective, two-parallel, and randomized study was approved by the Ethics Committee of Children's Hospital of Hebei Province (No. 129). The study was conducted in accordance with the Declaration of Helsinki (as revised in 2013). 

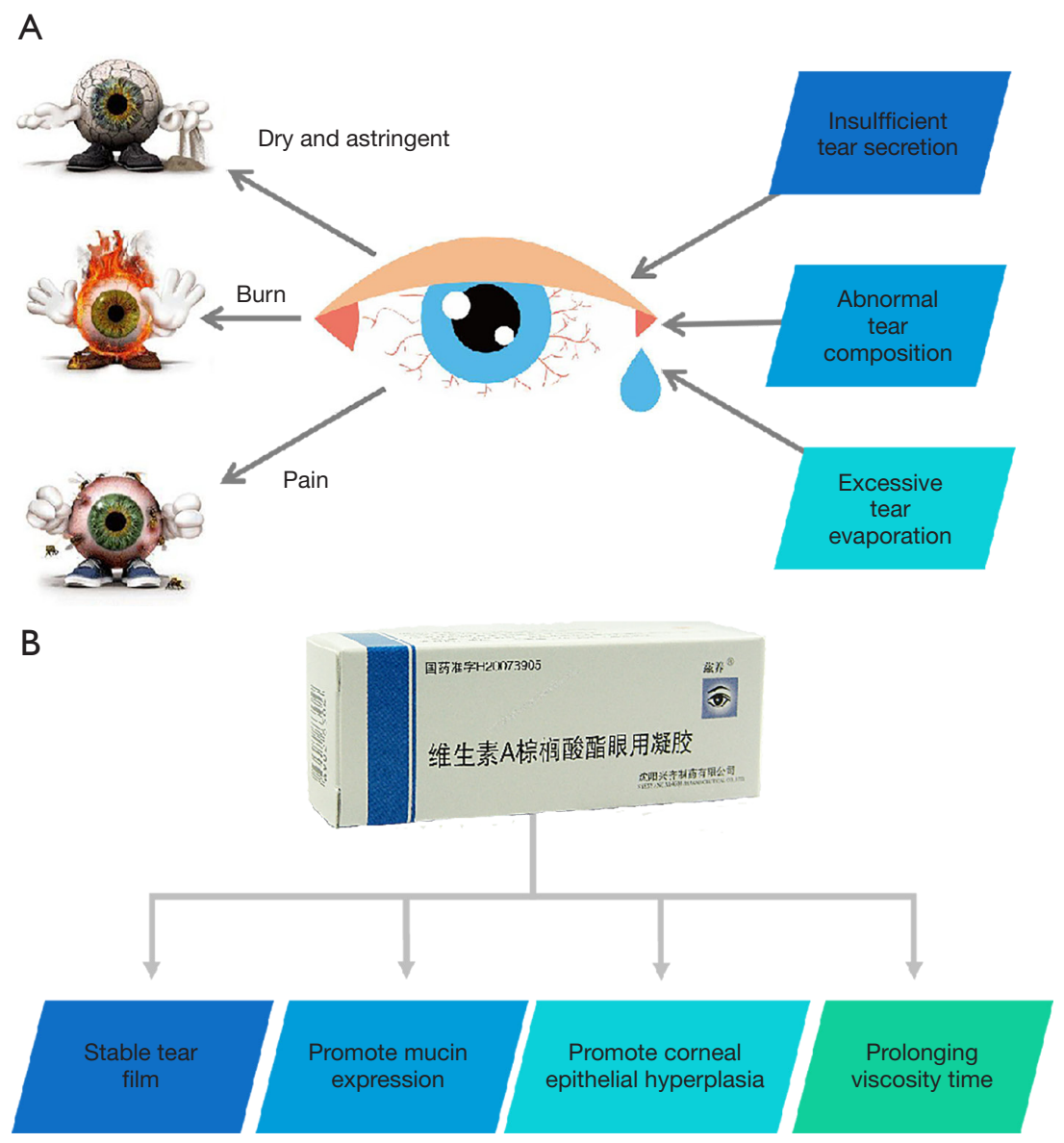

Figure 1 Symptoms and treatment of dry eye. (A) Symptoms of dry eye. (B) Mechanism of VAP eye gel. VAP, vitamin A palmitate.

The inclusion criteria were as follows: (I) dry eye diagnosed according to the criteria in "Dry eye syndrome preferred practice pattern ${ }^{\circledR}$ (13); (II) post-strabismus correction; (III) age range: $7-10$ years; and (IV) cases where clinical cooperation could be combined with various clinical examinations. The exclusion criteria were as follows: (I) cases involving long-term use of orthokeratology; (II) patients with concurrent eye infection, such as keratitis or conjunctivitis; (III) those with a history of eye surgery; and (IV) patients with systemic lupus erythematosus (SLE), rheumatoid arthritis (RA), or Sjogren's syndrome.

\section{VAP eye gel}

Vitamin A deficiency is an important inducing factor for dry eye. Long term lack of vitamin A can cause lacrimal gland atrophy, reduce tear secretion, and cause eye surface dryness, burning pain, and other symptoms (Figure 1A). In this study, VAP eye gel (H20073905, Shenyang Xingqi
Ophthalmic Co., Ltd., Shenyang, China) was used. Firstly, vitamin A supplementation can promote the proliferation and repair of cornea conjunctival epithelial cells, increase the expression of mucin, and improve the symptoms of corneal and conjunctival dehydration. Moreover, it increases the absorption of vitamin A by combining with palmitate gel, and produces a moisturizing and lubricating effect on the ocular surface (Figure 1B), thereby further enhancing the therapeutic effect.

\section{Study methods}

\section{Physicochemical property test}

For physicochemical property test, the size of VAP particles was evaluated under scanning electron microscopy. To test the solubility of VAP eye gel, these particles were evaluated in phosphate buffered saline (PBS) (10010002, ThermoFisher Scientific, MA, USA) and chloroform at 
$26^{\circ} \mathrm{C}$, and were observed every 5 minutes. The stability of these particles was test by evaluating the diameter alteration in chloroform in biochemical incubator (LBI-80, Shanghai Longyue Instrument Equipment Co., Ltd., China), which could fine control the temperature. We observed the diameters of VAP particles at $0-5,5-10,10-15,15-20$, and $20-25{ }^{\circ} \mathrm{C}$ respectively, so as to determine the best storage temperature, and these particles were observed every 5 minutes.

\section{Conjunctival goblet cells (GCs) culture}

In this study, we chose mouse conjunctival GCs for in vitro experiments, conjunctival GCs were dissociated and cultured using a protocol published by García-Posadas et al. (14). The cultured cells were diluted to $1 \times 10^{6}$ cells $/ \mathrm{mL}$, and evenly added to $24-w e l l$ plates. These cells were randomly divided into control group, carbomer group, and VAP group, VAP gel and carbomer gel were added into VAP and carbomer group respectively, then fetal bovine serum (FBS, 10\%, F8318, Sigma-Aldrich, St. Louis, MO, USA), 2 mM L-glutamine (G7513, Merck, Darmstadt, Germany), $100 \mu \mathrm{g} / \mathrm{mL}$ penicillin-streptomycin (V900929, Merck) were added to 24-well plates. The above cells were cultured in cell incubator (51032124, ThermoFisher Scientific) at $37^{\circ} \mathrm{C}$ and $5 \% \mathrm{CO}_{2}$ for another $24 \mathrm{~h}$.

\section{Conjunctival GCs activity detection in vitro}

Conjunctival GCs activity detection would be performed by immunofluorescence (IF). Anti-ki67 (ab15580, Abcam, USA) was used to label the active cells, and cell ability was observed by confocal microscope (FV3000, OLYMPUS, Japan). After 3 times of cleaning with PBS, the cells cultured above were fixed with $3 \%$ glutaraldehyde for 30 minutes, and were observed by confocal microscope.

\section{Group and treatment plan design}

According to the randomized digital table, 240 children with strabismus were divided into control and observation groups in the allocation ratio of $1: 1$, with 120 cases in each group. In the process of treatment and rehabilitation, VAP eye gel was additionally used in the observation group and Watson's care was simultaneously provided, while in the control group, routine nursing was provided to the children.

\section{Watson's care implementation plan}

A Watson's nursing care group was established, headed by the director of the nursing department. The group members included one head nurse, three nurses in charge, and five nurses. The director of the nursing department regularly gave lectures on Watson's theory of care, including topics such as a people-oriented nursing concept, the instilling trust and hope into patients, pacifying patients' emotions, and so on, in order to alter the service concept of nursing staff and improve nursing quality. In addition to theoretical teaching, clinical practice was carried out to enable nurses to better grasp the core of Watson's theory of care, and fully display this theory in every aspect of nursing.

Establishment of a good environment was important for the emotional state of the children, as children are more likely to be panicked when they first arrive in a strange environment, especially in a hospital with medical staff. Fear that is not eliminated in a timely manner could have a significant adverse impact on the follow-up treatment compliance of the children, as well as the clinical effect. As a result, a play room was set up in the ward, including an amusement park, comic book house, animated films, and so on. This would allow the children to eliminate their strangeness to the hospital while playing and increase familiarity between the children, thereby helping each other to eliminate panic. At the same time, the walls in the ward were painted with warm color, and the ward had sufficient light conditions, so as to create a good hospital environment for children. By improving the hospital environment, a strong humanistic care atmosphere can be fully reflected.

A lack of knowledge about dry eye among the children and their parents might affect their judgment of disease severity and cause unnecessary anxiety. A full account of the children's ages and the parents' cultural backgrounds was taken in order to implement personalized health education, and PPT, video, brochures, or games were used to explain the causes, symptoms, treatment methods, and prognosis of dry eye, in order to enrich the patients' understanding of dry eye, reduce their fears, and increase treatment compliance.

Psychological care is an important part of humanistic care. Understanding the negative emotions and timely counseling could effectively improve the treatment compliance of children. Nurses involved should adapt the concept of their roles, help children to receive medical treatment, promptly listen to their voices, and pay attention to their emotional changes, thus allowing for better understanding of their mentality, improving acceptance of surgical treatment, and enhancing their confidence to overcome the disease.

Sleep care also played an important role in the treatment of the children; sufficient rest played an important role in 
their physical recovery, maintaining a good mental state, and positive emotions. After admission, nursing staff worked to fully understand the children's work and rest rules with their parents, and attempted not to break their original work and rest routine, so as not to affect their biological clocks. At the same time, strong shade curtains were installed in the room to reduce the stimulation of sound and light during the rest period of children. Furthermore, the period of deep sleep was utilized for ward rounds, so as to avoid non-medical noise.

During hospitalization, the nurses paid attention to the safety protection work; for example, the table corners, stool feet, and the protruding parts of the wall were protected with anti-collision pads, and fences were installed beside the bed to avoid the occurrence of falls. At the same time, the nursing staff patrolled the ward every hour to reduce the occurrence of adverse safety events.

The treatment course of dry eye was long, and treatment was mainly conducted outside the hospital, and therefore, extended nursing outside the hospital was vital for the recovery of the children's condition. We utilized WeChat groups and telephone follow-ups, and nurses timely tracked the recovery degree of the children's condition. According to the degree of each child's condition and examination results, the nurses set up special care programs for their adjustment, and an eye massage video was sent on the WeChat group so that parents could instruct their children in rehabilitation exercises. At the same time, nurses adjusted and guided the children to eat more vitamin A-rich foods outside the hospital, rest, use VAP gel regularly, and avoid excessive eye use. In order to better guide the treatment of children, nurses promptly followed-up to ascertain the eye recovery status of children, and reorganize the review results into electronic documents.

\section{Assessment tools and methods}

(I) A self-designed treatment compliance adjustment questionnaire was used to record the treatment compliance of children, including regular medication use, eye hygiene, good eye habits, reasonable diet, and regular work and rest. To measure the treatment compliance of these five aspects, the following scoring system was used: complete compliance, 4 points; basic compliance, 3 points; occasional compliance, 2 points; and non-compliance, 1 point. Treatment compliance was considered good if score was $\geq 3$ points.

(II) Clinical efficacy evaluation: tear secretion was detected by the Schirmer I test (SIT) test. One end of the filter paper was folded back $5 \mathrm{~mm}$ and placed in the $1 / 3$ inside and outside of the conjunctival sac of the affected eye. Subsequently, the eyes were gently closed for $5 \mathrm{~min}$, the wetted length of the test paper was measured three times at 5 min intervals, and the final average value was recorded. The tear film break-up time (BUT) test was used to detect tear film stability. The sodium fluorescein test paper was moistened with ofloxacin eye drops and dipped in the conjunctival sac. After blinking 3-4 times, the fluorescein was evenly distributed on the eye surface. When the patient's eyes opened after the last eye closure, a slit lamp cobalt blue filter was used to observe the first black spot appearing on the cornea. The time was recorded and measured three times at 5 min intervals, and the average value was recorded. The integrity of corneal epithelium was detected by the same method as the BUT experiment. Fluorescent staining (FL) score of the cornea was observed using a slit lamp cobalt blue filter, and the staining degree was evaluated by the four-grade scoring method. The above tests were carried out before treatment, and at 1 and 3 months after treatment.

(III) Detection of inflammatory factors: at the first month after treatment, the sterilized and dried filter paper was placed on the inside of the affected eyelid fornix conjunctiva. After $5 \mathrm{~min}$, the filter paper was put into an EP tube containing $2 \mathrm{~mL}$ normal saline, and the samples were stored at $-80{ }^{\circ} \mathrm{C}$ for testing. The levels of IL- $1 \beta$, IL- 6 , and TNF- $\alpha$ in tears were detected by enzyme-linked immunosorbent assay (ELISA) and compared with the above indicators in tears before treatment.

(IV) The total effective rate was evaluated according to the symptoms after treatment and the results of SIT, BUT, and FL score, and was divided into four grades: clinical cure, markedly effective, effective, and ineffective.

(V) A self-designed satisfaction scale was used to assess family satisfaction, which was mainly evaluated in terms of service attitude, surgical quality, psychological intervention, medical environment, and ward management. Each index was scored at level 4, with higher scores indicating greater patient satisfaction.

\section{Statistical analysis}

SPSS18.0 statistical software package (SPSS, Chicago, 


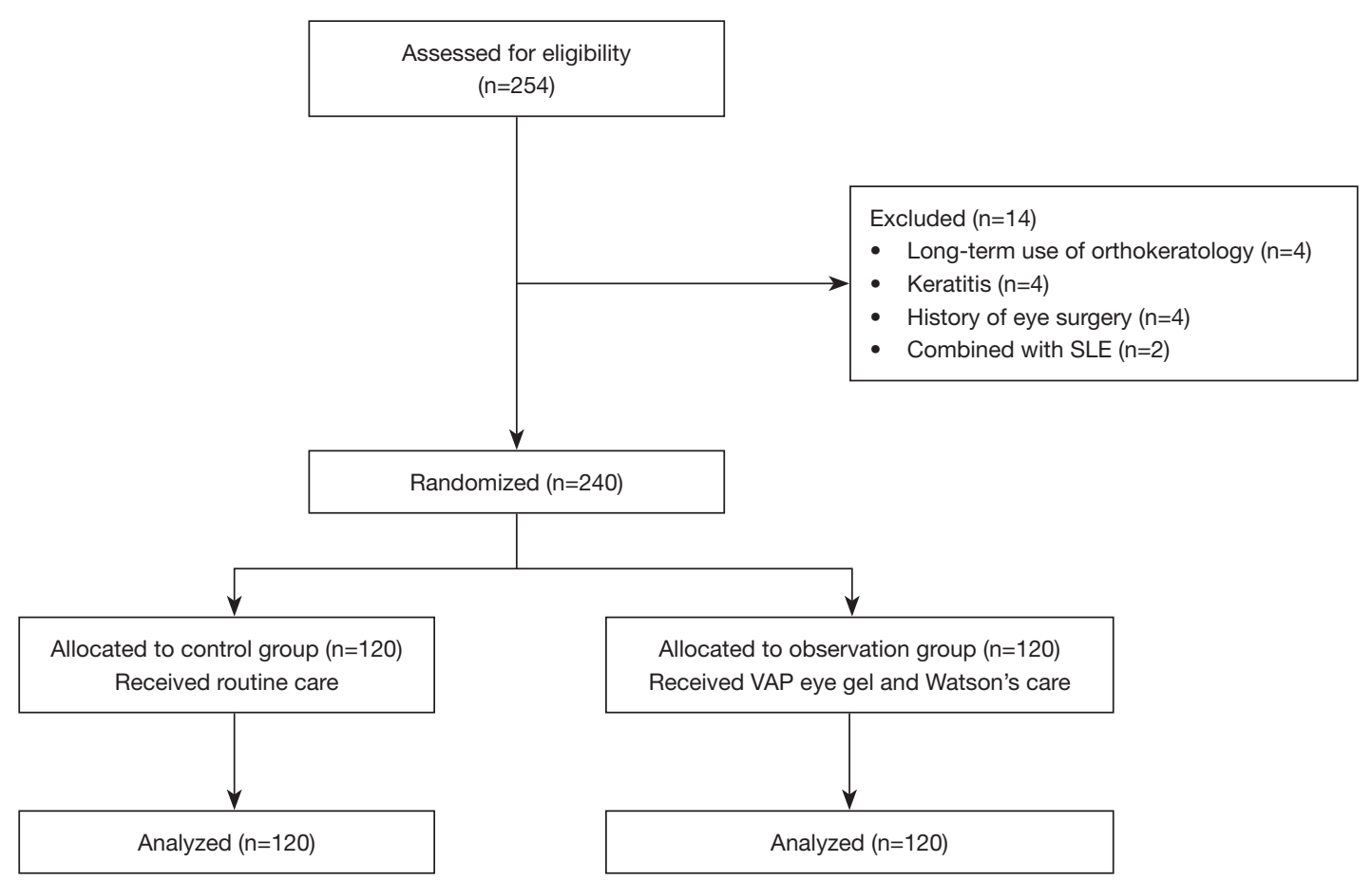

Figure 2 The CONSORT flow diagram of study participants. CONSORT, Consolidated Standards of Reporting Trials; SLE, systemic lupus erythematosus; VAP, vitamin A palmitate.

IL, USA) was used for data analysis, descriptive statistical analysis, and non-parametric test analysis. $\mathrm{P}<0.05$ was considered statistically significant.

\section{Results}

\section{General data}

From September 2018 to September 2020, 254 patients were assessed as qualified and 14 patients were excluded. These 14 patients consisted of 4 patients with long-term use of orthokeratology, 4 patients with keratitis, 4 patients with previous eye surgery and 3 patients with SLE. The remaining 240 patients were randomly divided into control group $(\mathrm{n}=120)$ and observation group $(\mathrm{n}=120)$ in the allocation ratio of 1:1. The Consolidated Standards of Reporting Trials (CONSORT) diagram was shown in Figure 2. No significant difference was found in age, sex, course of disease, BMI, diopter, and dry eye degree between the two groups $(\mathrm{P}>0.05$, Table 1).

\section{Physicochemical property of VAP}

In order to better promote the penetration of vitamin A into conjunctiva and corneal tissue, the good adhesion of eye gel can significantly improve the local absorption efficiency of vitamin $\mathrm{A}$. The study found that VAP eye gel was insoluble in water and could maintain good stability in PBS (Figure $3 A$ ). Therefore, VAP eye gel can better adhere to the eye and promote the absorption of vitamin A in the eyes. In addition, in order to better preserve VAP eye gel and explore its storage temperature, we discussed its solubility at different temperatures. The study found that after exceeding $20^{\circ} \mathrm{C}$, the solubility of VAP eye gel increased significantly, and it could maintain good coagulability at $0-5{ }^{\circ} \mathrm{C}$ (Figure $3 B$ ). Therefore, it is recommended to store it in refrigerators at $0-5{ }^{\circ} \mathrm{C}$ after opening, so as to maintain its good efficacy.

\section{GCs activity evaluation in three groups}

The injury of GCs in conjunctival epithelium will reduce the secretion of mucin, which is an important cause of dry eye. Therefore, promoting GCs proliferation is of great significance for the treatment of dry eye. The study found that the fluorescence intensity of GCs cultured in VAP gel and carbomer gel was significantly higher than that in pure medium, especially in VAP gel $(\mathrm{P}<0.05$, Figure 4$)$, 
Table 1 Comparison of baseline data between the two groups [n (\%); $\bar{x} \pm \mathrm{s}]$

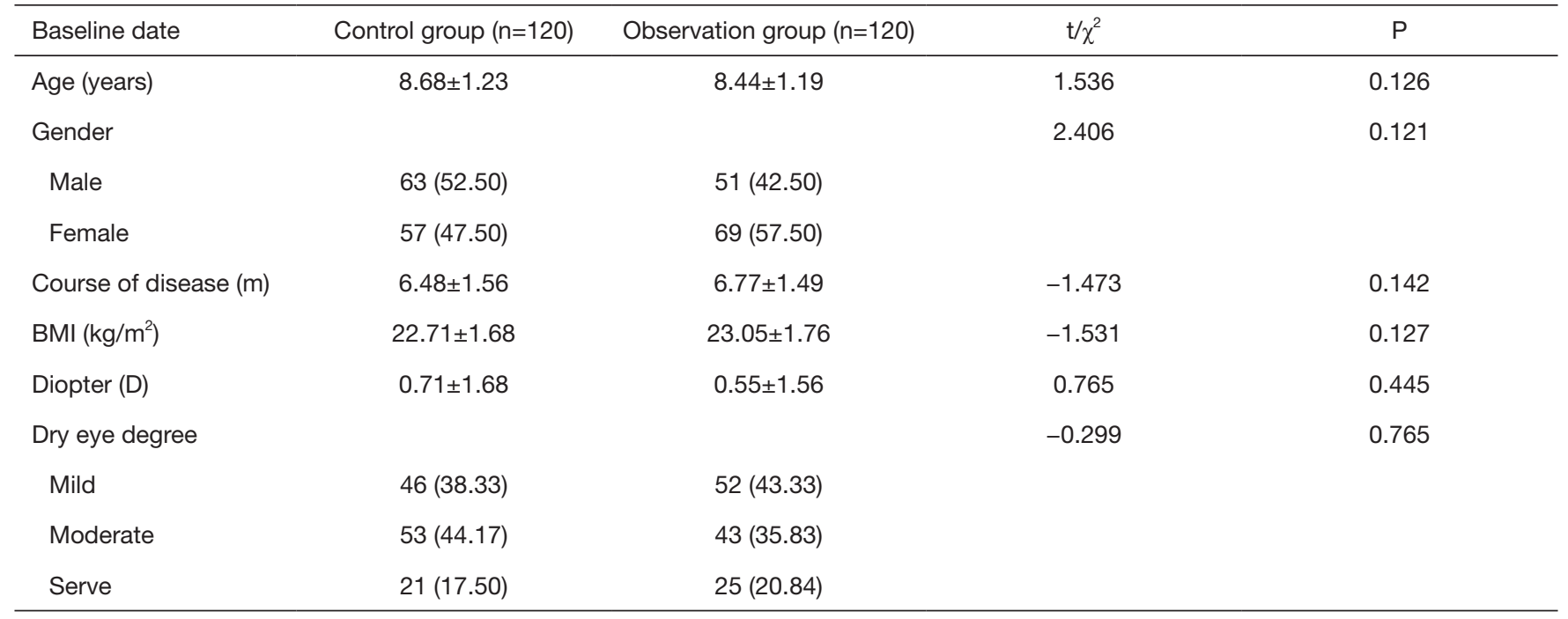
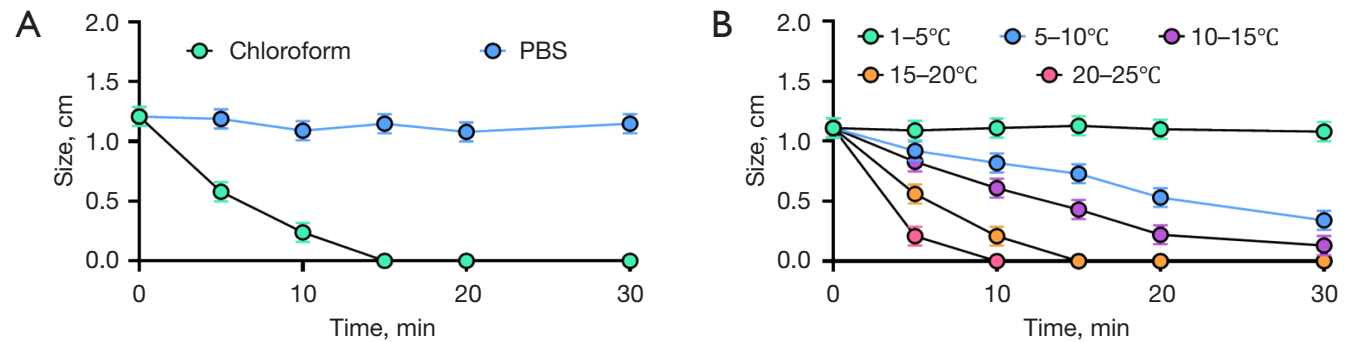

Figure 3 Solubility and stability detection. (A) Solubility test of VAP particles in PBS and vegetable oil. (B) Stability test of VAP particles at different temperatures. VAP, vitamin A palmitate; PBS, phosphate buffered saline.
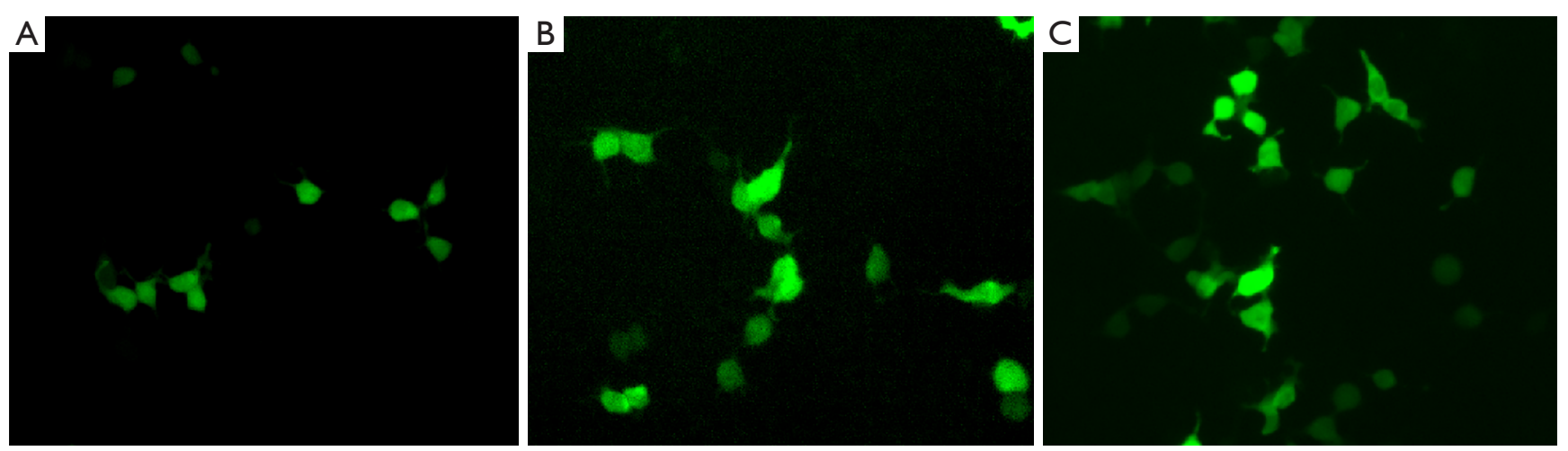

Figure 4 GCs activity assessment. (A) Anti-Ki67 expression pattern in control group. (B) Anti-Ki67 expression pattern in carbomer gel group. (C) Anti-Ki67 expression pattern in VAP group. IF staining, anti-ki67 (1:50), 10×. GCs, goblet cells; VAP, vitamin A palmitate; IF, immunofluorescence. 
suggesting that the addition of VAP gel could further improve the proliferation activity of GCs.

\section{Treatment compliance during the clinical treatment and nursing process}

The effect of treatment compliance played a significant role on the clinical effect of children with dry eye. This study evaluated the treatment compliance of children during the treatment process in terms of regular medication use, eye hygiene, good eye habits, reasonable diet, as well as regular work and rest. After the statistical questionnaire, we found

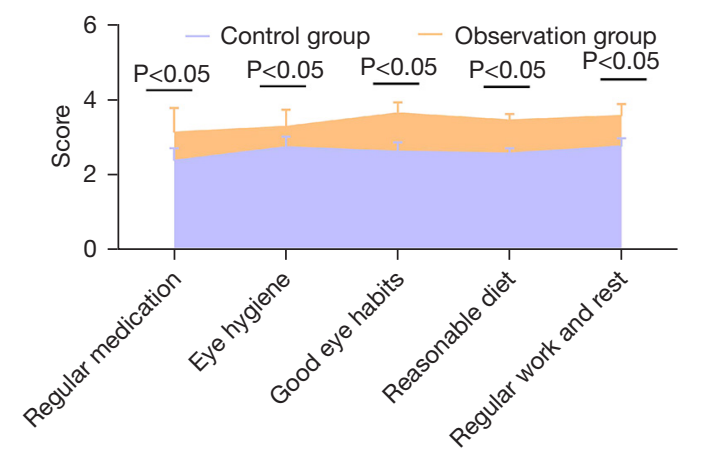

Figure 5 Comparison of treatment compliance in terms of regular medication use, eye hygiene, good eye habits, reasonable diet, regular work, and rest.

A
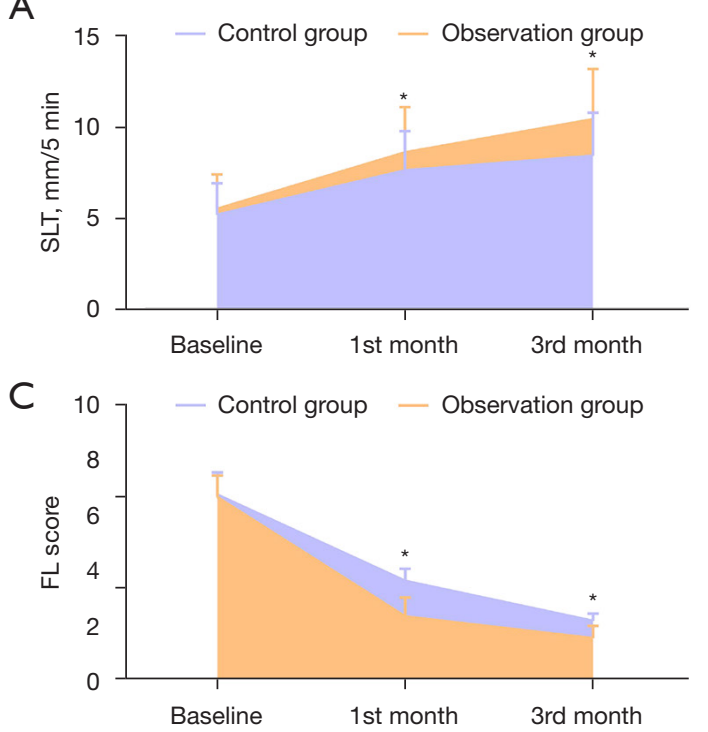

that the treatment compliance of observation group was higher than that of the control group $(\mathrm{P}<0.05$, Figure 5).

\section{Changes in SIT, BUT, and FL score}

The recovery of lacrimal gland secretion function can significantly reflect the clinical efficacy of dry eye treatment, and lacrimal gland function is detected by SIT. At 1 and 3 months post-treatment, the SIT increased significantly in both groups, especially in the observation group $(\mathrm{P}<0.05$, Figure $6 \mathrm{~A}$ ), which indicated better recovery of lacrimal gland secretion function.

Tear film stability can reflect the degree of destruction of conjunctival GCs, and its stability is detected by BUT. We found that the BUT increased significantly in both groups, especially in the observation group at 1 and 3 months after treatment $(\mathrm{P}<0.05$, Figure $6 B)$, which indicated better conjunctival recovery.

Also, dry eye patients lose the wetting effect of tears, and thus, the corneal epithelium is prone to friction damage, aggravation from foreign body sensation, photophobia, and pain. As a result, detection of corneal epithelial integrity can reflect the recovery of dry eye. Our results showed that the FL scores in the control group were higher at 1 and 3 months after treatment $(\mathrm{P}<0.05$, Figure $6 C)$, which indicated the corneal epithelium was defective, and the epithelial injury was more serious in control group

\section{B}

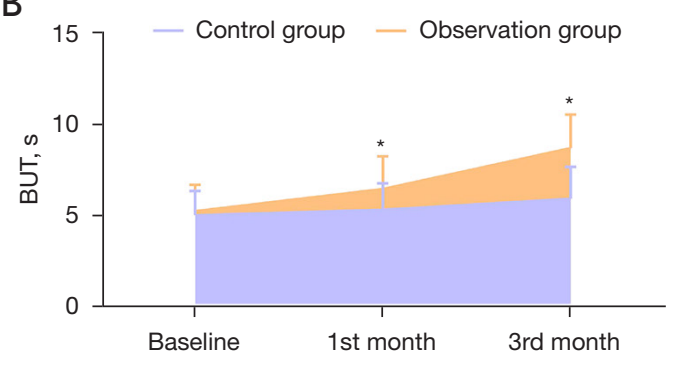

Figure 6 Comparison of clinical effect in terms of SIT (A), BUT (B), and FL score (C) at 1 and 3 months after treatment and clinical nursing $\left.{ }^{*}, \mathrm{P}<0.05\right)$. SIT, Schirmer I test; BUT, tear film break-up time; FL, fluorescent staining. 
A

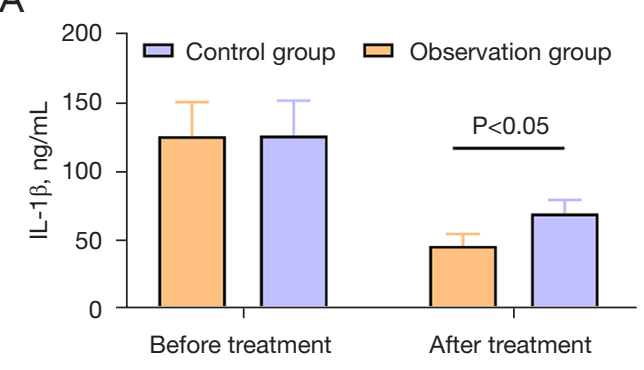

C

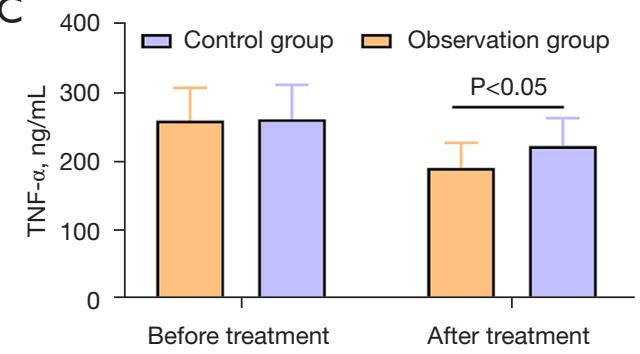

B

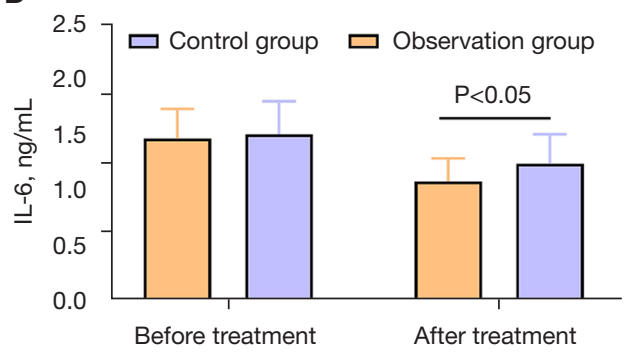

Figure 7 Comparison of IL-1 $\beta(\mathrm{A})$, IL-6 (B), and TNF- $\alpha(\mathrm{C})$ in tears between the two groups after treatment and rehabilitation training.

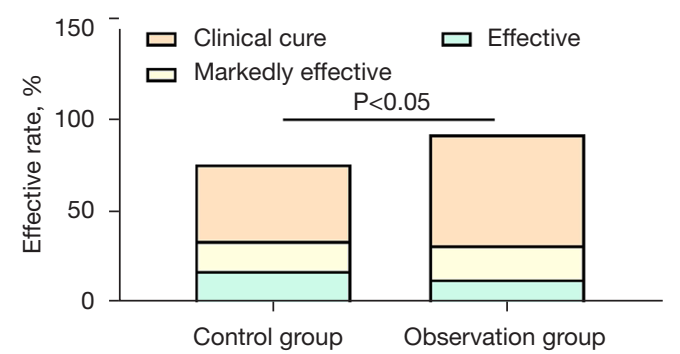

Figure 8 Comparison of effective rate of clinical treatment after treatment and clinical nursing.

\section{Changes in $I L-1 \beta, I L-6$, and TNF- $\alpha$ levels in tears after treatment and nursing}

Dry eye promotes the release of inflammatory factors into tears, leading to the occurrence of inflammatory reactions and oxidative damage to the eyes. As a result, the detection of inflammatory factors in tears was helpful in evaluating the severity of dry eye. After treatment, the levels of IL-1 $\beta$, IL6 , and TNF- $\alpha$ in the children's tears decreased, especially in the observation group $(\mathrm{P}<0.05$, Figure 7$)$, which indicated that the inflammation of the eyes decreased significantly.

\section{Effective rate of clinical treatment}

Based on the remission of the patients' eye symptoms and the slit lamp examination results, the treatment effect of dry eye was divided into four grades: clinical cure, markedly effective, effective, and ineffective. We found that after treatment and Watson's care nursing, the total effective rate of the observation group was improved more obviously $(\mathrm{P}<0.05$, Figure 8$)$, suggesting that the clinical treatment effect was better.

\section{Satisfaction of family members with nursing}

Nursing satisfaction was the most accurate evaluation of nursing effect. This study evaluated the service quality of nursing staff from in terms of service attitude, quality of treatment, psychological intervention, medical environment, and ward management. The questionnaire survey showed that the service scores of the observation group were significantly higher $(\mathrm{P}<0.05$, Figure 9$)$, indicating greater nursing satisfaction in this group.

\section{Discussion}

Nurses play an important role in the treatment of children with dry eye; they not only perform clinical nursing work, but also play an important role in enhancing the child's mood, mentality, and safety. As a special medical group, nursing staff should pay close attention to children's physical diseases as well as their psychological reactions to the treatment process (15). In the process of child care, "people- 


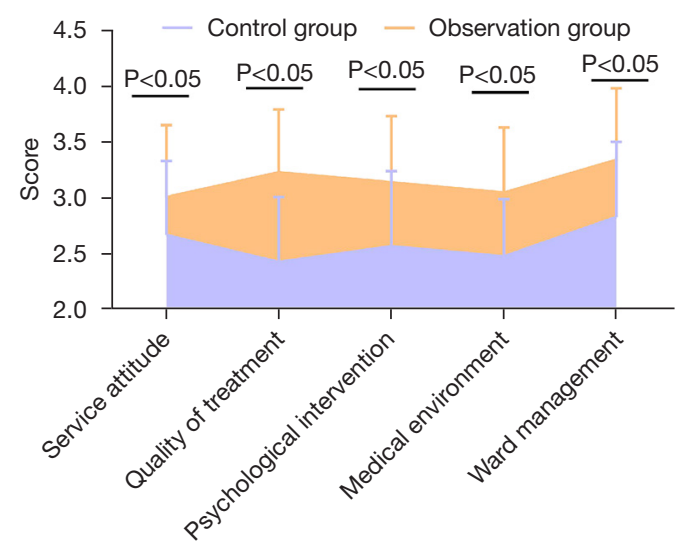

Figure 9 Comparison of satisfaction degree in terms of service attitude, quality of treatment, psychological intervention, medical environment, and ward management.

oriented" is the core concept of nursing, which involves more patience and care (16), and will help to improve the treatment compliance of children and facilitate the building of a harmonious relationship between nurses and patients. Watson's theory posits that nursing work is a discipline of nurses' sincerity, care, and love (17). During the provision of nursing service, comprehensively reflecting humanistic care is crucial for nurses in order to obtain a sense of professional identity, and is an indispensable structural factor in high-quality nursing. Therefore, enacting Watson's theory training for nursing staff is important in improving the quality of medical care.

VAP is mainly composed of vitamin A, Carbomer and palmitate. As the main ingredients, vitamin A can prevent keratosis of cornea and conjunctival epithelium, and promote GCs to secrete mucin, thus effectively wetting the ocular surface and avoiding the invasion of pathogenic bacteria. Carbomer, as a substrate of vitamin A, can form a liquid reservoir inside the gel after acting on the ocular surface. It can continuously release moisture in the blink process, so as to better wet the eyes and increase the stability of tear film. The combination of vitamin A and carbomer can increase the stability of vitamin A and release drugs slowly and permanently. The application of palmitate can further maintain the integrity of the eye lipid layer and reduce the evaporation of tears, thus better improving the symptoms of dry eyes. Compared with the pure carbomer gel, VAP can effectively supplement vitamin A, thereby promoting the proliferation of GCs and mucus secretion (18). It could maintain the balance of lipid layer, water sample layer and mucus layer in the tear film, so as to better maintain the stability of tear film and make the eyes better moisten with tears.

The treatment cycle of dry eye is long, and most of the treatment process is conducted outside the hospital. As a result, the treatment compliance of children and their families plays a significant role in the prognosis of disease (19). In order to quantitatively evaluate the treatment compliance of children during the treatment period, we assessed their regular medication use, eye hygiene, good eye habits, reasonable diet, as well as regular work and rest. Following Watson's theory of care guidance and training, nursing staff paid attention to the details of the nursing process from the perspective of children and their families, so as to improve the patient experience and nursing quality. For example, diversified modes were adopted to carry out personalized health education, wards were painted with a warm color, a children's paradise was set up, more attention was promptly paid to the psychological state of children, and a quiet and comfortable sleeping environment was built. Considering that treatment compliance is more easy declined in a nonhospital setting, nursing staff were specially arranged to carry out extended remote nursing guidance through WeChat, Internet, telephone, and other measures, and provided supervision and guidance in regular medication use, reasonable diet, regular work, and rest. Using Watson's care nursing, the people-oriented concept was implemented in the entire treatment and recovery process, and thus, the quality of treatment compliance in the observation group was markedly improved.

In addition to the subjective feelings of patients, the evaluation criteria for the efficacy of dry eye primarily included the amount of tear secretion, tear film stability, and corneal epithelial integrity (20). The treatment compliance of the observation group was significantly improved, and the children could better follow the doctor's treatment advice, including massaging the eye points to promote tear secretion, regular use of VAP eye gel, diet to supplement vitamin A intake, ensuring eye hygiene, etc. As a result, the treatment effect was also notably improved. Specifically, the use of VAP eye gel, which is rich in vitamin $\mathrm{A}$, is an important element in the differentiation, proliferation, and repair of corneal epithelial cells and conjunctival GCs (21), thereby preventing squamous conformation of the conjunctival epithelium. At 1 month after treatment, we observed that the corneal epithelial injury recovery degree of the observation group was better, and its FL score was lower.

In addition to the normal ocular surface structure, a stable tear film is also critical for the recovery of dry eye. Vitamin A 
can promote the expression of ocular surface mucin (22), and through the combination with palmitate, the water retention effect can be increased, resulting in a significantly prolonged viscosity time (23). Also, the absorption of vitamin A in the lipid will be promoted, thereby encouraging the recovery of tear film stability (24). Therefore, at 1 and 3 months after treatment, the BUT time recovery of children in the observation group was better, which could make tears evenly distributed on the ocular surface and significantly promote the secretion function of conjunctival GCs, resulting in increased tear secretion. In addition, the injury of corneal and conjunctival epithelial cells, and local inflammatory reaction, can lead to increased levels of inflammatory factors in tears. VAP eye gel can promote corneal epithelial repair and stabilize the tear film, and as a result, inflammation levels in tears decreased more obviously in the observation group. In general, the total effective rate of the observation group was higher after treatment. This group also benefited from the nurses' more thoughtful care; establishing of a helpful and trusting relationship with children and their families helped the children to reduce negative emotions, which significantly improved the parents' satisfaction. Moreover, this could further enhance the medical relationship and build a harmonious hospital.

\section{Conclusions}

In this study, through Watson's care course training for nursing staff, the "people-oriented" concept of nursing staff was effectively improved. In the process of Watson's care, nurses focus on children and care for them wholeheartedly, which effectively improved the children's treatment compliance. At the same time, the use of VAP gel can aid the proliferation of conjunctival GCs, and stabilize the tear film structure, which can enhance the clinical efficacy. Therefore, VAP eye gel combined with Watson's theory of care play an important role in reducing the occurrence of dry eye after strabismus surgery, in addition, it could effectively improve patients' satisfaction and the relationship between nurses and patients, which was helpful to build a harmonious hospital.

\section{Acknowledgments}

Funding: Scientific research projects funded by Hebei Provincial Health Commission: Study on the Influence of Nursing Quality and Satisfaction of Strabismus Surgery Patients Based on Watson Care Theory (No: 20211435).

\section{Footnote}

Reporting Checklist: The authors have completed the CONSORT reporting checklist. Available at https://dx.doi. org/10.21037/tp-21-385

Trial Protocol: Available at https://dx.doi.org/10.21037/tp21-385

Data Sharing Statement: Available at https://dx.doi. org/10.21037/tp-21-385

Conflicts of Interest: All authors have completed the ICMJE uniform disclosure form (available at https://dx.doi. org/10.21037/tp-21-385). The authors have no conflicts of interest to declare.

Ethical Statement: The authors are accountable for all aspects of the work in ensuring that questions related to the accuracy or integrity of any part of the work are appropriately investigated and resolved. The study was conducted in accordance with the Declaration of Helsinki (as revised in 2013). All children and their guardians who participated in the study gave informed consent to the study and voluntarily signed the informed consent. This study was approved by the Ethics Committee of Children's Hospital of Hebei Province (No. 129).

Open Access Statement: This is an Open Access article distributed in accordance with the Creative Commons Attribution-NonCommercial-NoDerivs 4.0 International License (CC BY-NC-ND 4.0), which permits the noncommercial replication and distribution of the article with the strict proviso that no changes or edits are made and the original work is properly cited (including links to both the formal publication through the relevant DOI and the license). See: https://creativecommons.org/licenses/by-nc-nd/4.0/.

\section{References}

1. Kato K, Miyake K, Hirano K, et al. Management of postoperative inflammation and dry eye after cataract surgery. Cornea 2019;38 Suppl 1:S25-33.

2. O'Neil EC, Henderson M, Massaro-Giordano M, et al. Advances in dry eye disease treatment. Curr Opin Ophthalmol 2019;30:166-78.

3. Tang J, Hallam RA, Francis J, et al. Exploring the relationship between quality rating and improvement 
system supports and global quality in family child care. Child \& Youth Care Forum 2020;49:893-914.

4. Ing MR, Shortell J, Golez J. Extraocular and intraocular infections following strabismus surgery: a review. J Pediatr Ophthalmol Strabismus 2019;56:214-21.

5. Sim PY, Cleland C, Dominic J, et al. Investigation of factors associated with the success of adult strabismus surgery from the patient's perspective. J AAPOS 2018;22:266-271.e3.

6. Hooper A, Schweiker C. Prevalence and predictors of expulsion in home-based child care settings. Infant Ment Health J 2020;41:411-25.

7. Naderi K, Gormley J, O'Brart D. Cataract surgery and dry eye disease: a review. Eur J Ophthalmol 2020;30:840-55.

8. Welyczko N. Healthcare Simulation Education: Evidence, Theory and Practice Nestel Debra Kelly Michelle Jolly Brian Watson Marcus Healthcare Simulation Education: Evidence, Theory and Practice 249pp £44.99 Wiley Blackwell 9781119061595 1119061598. Nurs Stand 2017;31:33.

9. Fantin M, Buzzacaro E, Aguiar DCM, et al. Patientsâ€ $€^{\mathrm{TM}}$ perception in hospital environment: a look at nursing care in perioperative period based on Jean Watson conceptions. Scientific Electronic Archives 2019;12:102-9.

10. Chiang FW, Chang JL, Hsu SC, et al. Dexmedetomidine use in pediatric strabismus surgery: a systematic review and meta-analysis. PLoS One 2020;15:e240553.

11. Singer JR, Bakall B, Gordon GM, et al. Treatment of vitamin A deficiency retinopathy with sublingual vitamin A palmitate. Doc Ophthalmol 2016;132:137-45.

12. He W, Guo X, Feng $M$, et al. In vitro and in vivo studies on ocular vitamin A palmitate cationic liposomal in situ gels. Int J Pharm 2013;458:305-14.

13. Akpek EK, Amescua G, Farid M, et al. Dry eye syndrome preferred practice pattern ${ }^{\circledR}$. Ophthalmology 2019;126:P286-334.

14. García-Posadas L, Hodges RR, Diebold Y, et al. Contextdependent regulation of conjunctival goblet cell function by allergic mediators. Sci Rep 2018;8:12162.

15. Cohen L, Schwartz N, Guth A, et al. User survey of

Cite this article as: Yang S, Guo W, Gong Y, Wang J, Chen L, Zhao J, Guo X, Bai J, Song Y. Application of vitamin A palmitate eye gel and nurse value of Watson's theory of caring in children with dry eye after strabismus surgery: a randomized trial. Transl Pediatr 2021;10(9):2335-2346. doi: 10.21037/tp21-385
Nanny Angel Network, a free childcare service for mothers with cancer. Curr Oncol 2017;24:220-7.

16. Aktürk Ü, Erci B. The effect of Watson's human caring model on meaning of life and symptom management in cancer patients undergoing chemotherapy. Res Theory Nurs Pract 2018;32:255-75.

17. Erbay Ö, Yıldırım Y, Fadıloğlu Ç, et al. Use of Watson's theory of human caring in nursing care of patients with hypertensive attack: case report. Turkish J Cardiovasc Nurs 2018;9:82-8.

18. Cui X, Xiang J, Zhu W, et al. Vitamin A palmitate and carbomer gel protects the conjunctiva of patients with long-term prostaglandin analogs application. J Glaucoma 2016;25:487-92.

19. Szczotka-Flynn LB, Maguire MG, Ying GS, et al. Impact of dry eye on visual acuity and contrast sensitivity: dry eye assessment and management study. Optom Vis Sci 2019;96:387-96.

20. Rouen PA, White ML. Dry eye disease: prevalence, assessment, and management. Home Healthc Now 2018;36:74-83.

21. Qiu XD, Gong L, Chen MJ. Research on effects of vitamin A palmitate on repair of mechanical corneal epithelial defects and conjunctival goblet cells in rabbits. Zhonghua Yan Ke Za Zhi 2010;46:151-60.

22. Cui C, Li L, Li M. Improvement of lipase activity by synergistic immobilization on polyurethane and its application for large-scale synthesizing vitamin A palmitate. Prep Biochem Biotechnol 2019;49:485-92.

23. Berson EL, Weigel-DiFranco C, Rosner B, et al. Association of vitamin A supplementation with disease course in children with retinitis pigmentosa. JAMA Ophthalmol 2018;136:490-5.

24. Hodges JK, Tan L, Green MH, et al. Vitamin A supplementation redirects the flow of retinyl esters from peripheral to central organs of neonatal rats raised under vitamin A-marginal conditions. Am J Clin Nutr 2017;105:1110-21.

(English Language Editor: A. Kassem) 